\title{
Monitoring the Radiation Injury of Red Blood Cells to Micowave Radiation with Different Power Density
}

\author{
Junguang Yong ${ }^{1}$, Ping Ruan ${ }^{1}$, Hongtao Shen ${ }^{2}$ \\ ${ }^{1}$ Guangdong Pharmaceutical University, Guangzhou, China \\ ${ }^{2}$ College of Physics and Technology, Guangxi Normal University, Guilin, China \\ Email: Rp711@sina.com, shenht@ciae.ac.cn
}

Received 2013

\begin{abstract}
Multiple state-of-the-art techniques, such as multi-dimensional micro-imaging, fast multi-channel micro-spetrophotometry, and dynamic micro-imaging analysis, were used to dynamically investigate various effects of cell under the 900 $\mathrm{MHz}$ electromagnetic radiation. Cell changes in shape, size under different power density electromagnetic waves radiation were presented in this paper. Experimental results indicated that the isolated human red blood cells (RBCs) do not have obviously real-time responses to the ultra-low density $\left(15 \mu \mathrm{W} / \mathrm{cm}^{2}, 31 \mu \mathrm{W} / \mathrm{cm}^{2}\right)$ electromagnetic wave radiation when the radiation time is not more than 30 minutes; however, the cells do have significant reactions in shape, size to the electromagnetic waves radiation with power densities of $1 \mathrm{~mW} / \mathrm{cm}^{2}$ and $5 \mathrm{~mW} / \mathrm{cm}^{2}$. The data reveals the possible influences and statistical relationships among living human cell functions, radiation amount and exposure time with high-frequency electromagnetic waves. The results of this study may be significant on protection of human being and other living organisms against possible radiation affections of the high-frequency electromagnetic waves.
\end{abstract}

Keywords: Biomedical Measurement; RBCs; Electromagnetic (EM) Waves; Power Density

\section{Introduction}

As the modern electronics products become more and more popular, radiations from various appliances, such as consumer electronics, automated office equipment, high voltage transport lines, power stations etc., begin to be a serious human exposure issue with potential health consequences. These radiation waves can possibly penetrate various substances including human body cells and has become the fourth biggest pollution source subsequent to water contamination, air and noise pollutions. So far, there are a lot of investigations on biological responses to the EM (Electromagnetic) radiation. There is a wide range of data documenting the ability of EM radiation to affect the biochemical and molecular mechanisms of cells both in vitro and in vivo with effects independent of thermal phenomena such as, altered cell growth [1], exocytosis [2], gene expression [3], chromosomal instability [4] and the expression of heat shock proteins [5,6]. The effects of EM radiations on human body are generally accepted. $[7,8]$.

However, there are only scattered studies dealing with the influences of EM waves on RBCs geometric characteristics. In most previous studies the effects were generally measured after a relatively long period of radiation due to the limitations of experimental conditions. Due to the self-repairing process, however, the changes in cells may be recovered or distorted in the delayed observations. Hence, real-time research and measurements of EM waves radiation effects on cells should be carried out. In this work, the influences of EM radiation on live red cells geometric characteristics were studied with different power densities and different radiation time intervals.

\section{Materials and Methods}

1) Technology and Methods

The imaging and analysis system used in this research has multiple functions of multi-dimensional micro-imaging, fast multi-channel micro-spetrophotometry, and dynamic micro-imaging. It is able to measure multiple structures and parameters of live cells simultaneously and monitor the changes of molecule structures and substance concentration. The whole system, shown in Figure 1, is based on an inverted microscope (Nikon TE300, Tokyo, Japan). The prepared samples were mounted under the microscope, and a halogen lamp is used to produce light beams which go through the adjustable optics pinhole 1 and then focused onto the samples. The aperture of the optical pinhole can be adjusted in the range of $1 \mu \mathrm{m} \sim 200 \mu \mathrm{m}$. The transmitted light from the samples penetrates an objective lens and a transflective lens, and 


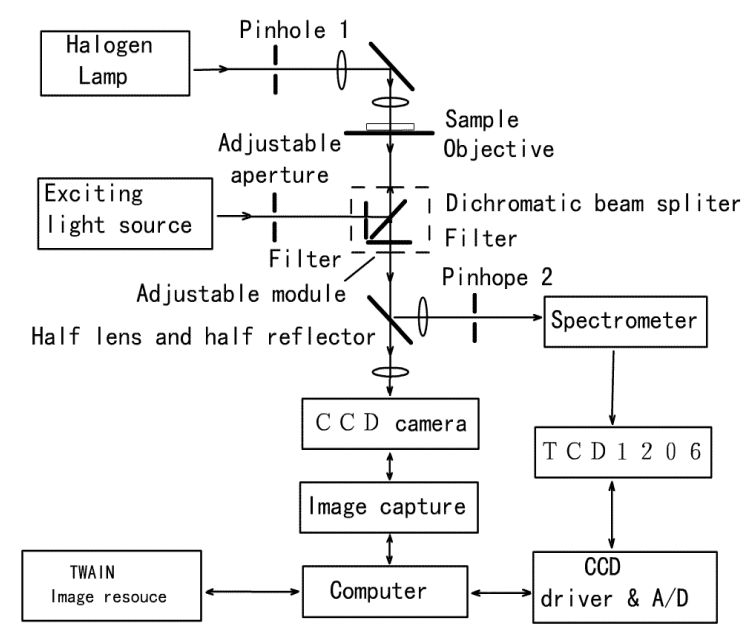

Figure 1. Experimental equipment.

goes through the image analytical system of microscopic organisms. A CCD (Charge-Coupled Device) camera (IMAC-CCDS30 $768 \times 572$, Tokyo, Japan) and an image capturing card (MATROX-METEOR II PCI, Dorval, Canada) are used to capture the microscopic images and send them to the system host (Lenovo ThinkStation D20, Beijing, China) for real-time image processing, analysis, storage and display.

The analytic system of microscopic images can capture the microscopic images of the RBCs and then automatically recognize and count the cells [9]. It also can give out quantitative information of single cell in shape, size and other characteristics. The image analysis includes capturing and buffering the selected cells images, binary conversion of the images with suitable thresholds, cell edge detection and separation. The analysis process also includes space location, measurement calibration, and calculation of cell contact area, perimeter, long axis, short axis, long-short axis ratio, normalized factors of shape and other mechanical parameters. Among these values, the long axis and short axis values are one dimensional, which represent the cell deformation; the contact area and perimeters are two dimensional, which represent the cell size. The normalized factors, between $0 \sim 1$, are geometric characteristics of RBCs.

2) EM Wave Exposure

The EM waves are radiated by a pillar antenna located outside of the plastic incubator. In our early experiments $[10,11]$, we have been using the same setup as exposure devices for getting the different power densities. The EM signal is generated by a EM wave generator VS-401A (Verition, Shenzheng,China) and firstly amplified by a linear Power Amplifier [Model PF0342A] before it is fed to the pillar antenna. The generator operates in the 900 $\mathrm{MHz}$ frequency (GSM standing waves, adopted by China Mobile,) with the frequency stability $\pm 0.05 \%$. The maximum output power of the generator is about $10 \mathrm{~mW} / \mathrm{cm}^{2}$.
A PMM-8053 EM analyzer combined with a PMMEP300 probe (PPM, Segrate, Italy) was used to monitor the power density and electric field strength of EM waves around the sample. The signal source output level can be adjusted through modifying the distance between antenna and cell pools. Four levels of power densities, two of which $\left(15 \mu \mathrm{W} / \mathrm{cm}^{2}, 31 \mu \mathrm{W} / \mathrm{cm}^{2}\right)$ corresponding to the radiation power of mobile phones and the other two (1 $\mathrm{mW} / \mathrm{cm}^{2}, 5 \mathrm{~mW} / \mathrm{cm}^{2}$ ) corresponding to mobile phone stations, microwave oven production lines as well as airport radar stations could be obtained by this method. Considering the cell pools are very small $(5 \mathrm{~mm} \times 5 \mathrm{~mm})$ with the distance from the signal source, the EM fields in the cell poolsare considered to be uniform. Absorbed power was calculated by an Ansoft HFSS simulation code. Using this method of calculation, an average incident power of $1.25 \mathrm{~mW}\left(5 \mathrm{~mW} / \mathrm{cm}^{2}\right)$ resulted in $0.26 \mathrm{~mW}$ of absorbed power. Thus approximately 21 percent of the incident power was absorbed by the sample. The $0.26 \mathrm{~mW}$ of absorbed power produced an average specific absorption rate (SAR) of $2.08 \mathrm{~W} / \mathrm{Kg}$, since the volume of the sample cell was 0.125 cc. By this method, the SARs for the four levels of power densities $15 \mu \mathrm{W} / \mathrm{cm}^{2}, 31 \mu \mathrm{W} / \mathrm{cm}^{2}, 1$ $\mathrm{mW} / \mathrm{cm}^{2}$ and $5 \mathrm{~mW} / \mathrm{cm}^{2}$ are $6.24 \mathrm{~mW} / \mathrm{kg}, 12.90 \mathrm{~mW} / \mathrm{kg}$, $0.42 \mathrm{~W} / \mathrm{kg}$ and $2.08 \mathrm{~W} / \mathrm{kg}$. All exposures were controlled with the assistance of a computer system and all exposure data, such as input power level of the antenna and the temperature inside exposure incubator (maintained at $37^{\circ} \mathrm{C}$ with the stability $\pm 0.1^{\circ} \mathrm{C}$ ) were checked every second to confirm that exposure conditions in the incubator were achieved as scheduled. The temperature time course was measured in preliminary tests by means of a fiber optic thermometer (Geokon BGK-FBG-4700, Beijing, China). Measured data showed that there is no increase of temperature observed at $5 \mathrm{~mW} / \mathrm{cm}^{2}, 15 \mu \mathrm{W} / \mathrm{cm}^{2}, 31$ $\mu \mathrm{W} / \mathrm{cm}^{2}$ and $1 \mathrm{~mW} / \mathrm{cm}^{2}$.

3) Preparation of Human Red Cell Samples

A pipe of intravenous blood was sampled from a healthy adult volunteer (who had signed a contract according the local law) after a medical examination. After adding parnaparin sodium (anticoagulants), the sample was centrifugalized at a speed of $2000 \mathrm{rpm}$ (revolutions per minute) for 10 minutes. The white cells and blood platelet in the upper layer were then removed. The remaining red cell solution was washed by 10 times of iso-osmotic PBS liquid in volume, and centrifugalized [12]. Three additional cycles of washing-centrifugation are needed for cleaning the cells. The cleaned cells were then put into glucosans liquids for further centrifugation. The density (g/g) of the glucosans liquids should be $23 \%, 22 \%, 21 \%, 20 \%$, $19 \%$, respectively in the following centrifugations. Each centrifugation is in a speed of $3000 \mathrm{rpm}$ (1198 g-force) for 40 minutes. By this way, "Young Red Cells" at top of the sample can be obtained and then put into iso-osmotic 
PBS liquid with the ratio1:8000 in volume [13]. In the subsequent measurement, the sample liquid was transferred onto the glass slides and put into a micro cell pools after the temperature reaches the preset value of $37^{\circ} \mathrm{C}$. The temperature was kept at $37^{\circ} \mathrm{C}$ during all the measurements.

\section{4) Experimental}

RBCs were continuously monitored and measured before, in the course of, and after radiation, respectively, in the following two experiments.

a) RBC samples were irradiated by $900 \mathrm{MHz}$ EM waves with power densities of $15 \mu \mathrm{W} / \mathrm{cm}^{2}$ (SAR 6.24 $\mathrm{mW} / \mathrm{kg}$ ), $31 \mu \mathrm{W} / \mathrm{cm}^{2}$ (SAR $12.90 \mathrm{~mW} / \mathrm{kg}$ ), $1 \mathrm{~mW} / \mathrm{cm}^{2}$ (SAR $0.42 \mathrm{~W} / \mathrm{kg}$ ) and $5 \mathrm{~mW} / \mathrm{cm}^{2}$ (SAR $2.08 \mathrm{~W} / \mathrm{kg}$ ), respectively, for 30 minutes each. The contact area, perimeter, long-short axis ratio, and normalized factors (a parameter describe the stiffness and deformability of cells in the software) of real-time geometric shaping for the cells were then measured with microscopic imaging and analysis. About 80 cells were measured for each sample in two seconds.

b) The EM wave with $900 \mathrm{MHz} 5 \mathrm{~mW} / \mathrm{cm}^{2}$ (SAR 2.08 $\mathrm{W} / \mathrm{kg}$ ) was used to irradiate the RBCs for 60 minutes, and the measurements were performed at the beginning, the tenth, the $20^{\text {th }}$ and the $30^{\text {th }}$ minute of irradiation and the $30^{\text {th }}$, the $60^{\text {th }}$ minute after radiation, respectively. The parameters determined include contact area, perimeter, long-short axis ratio and normalized factors, etc. About 80 cells were measured for each sample in two seconds.

5) Statistical Analysis 1

Cell changes in shape, size and parameters of $\mathrm{Hb}$ absorption spectrum under different power density electromagnetic waves radiation were measured. For estimation of the effect in each independent group, the averaged parameter was first calculated; the error of these calculations was less than $10 \%$. Student's t-test was used to compare different group of data. For statistical analysis we used the data obtained from different cells; the number of cells is about 80 . The level of statistical significance was basically set at 5\%. For statistical analysis, SPSS software (ver11.0, IBM, Chicago, USA) was employed.

\section{Results and Discussion}

As can be seen from Table 1, the geometric characteristics of the tested RBCs had no significant changes ( $\mathrm{P}>$ 0.05) after 30 minutes radiation of the ultra-low power irradiation $\left(15 \mu \mathrm{W} / \mathrm{cm}^{2}, 31 \mu \mathrm{W} / \mathrm{cm}^{2}\right)$ However, after 30 minute irradiation with power densities of $1 \mathrm{~mW} / \mathrm{cm}^{2}$ and $5 \mathrm{~mW} / \mathrm{cm}^{2}$, the size and the long to short axis ratio of RBCs decreased, and the normalized factors of RBCs increased $(\mathrm{P}<0.05)$. Part of RBCs was changed from double concaves to acanthocytes. As power density increases, the aggregation of red cells increases accordingly.

Real-time reactions of live RBCs to $900 \mathrm{MHz}$ EM wave with $5 \mathrm{~mW} / \mathrm{cm}^{2}$ power density are shown in Table $\mathbf{2}$, where the measurements were performed at different times in the course of and after radiation, respectively. As the radiation time increases, the contact area and perimeter of red cells become smaller while the long to short axis ratio and the normalized factors become larger compared to the corresponding values before radiation. As the time of radiation over the RBCs becomes longer, the number of the deformed RBCs increases. In fact, after 60 minutes of radiation, all RBCs were deformed to be acanthocytes. Aggregation was strengthened as more acanthocytes were formed. If the radiation time is longer than 60 minutes, this kind of deformation will be irreparable. Figure 2 shows the results of RBCs deformation under EM waves' radiation measured at different time points: before radiation, after 30 minutes and after $60 \mathrm{mi}-$ nutes radiation.

The red cells will be changed in shape, size, and su-

Table 1. Geometric parameters of RBC vs power density (for $900 \mathrm{MHz}$ EM wave) after 30 minutes $\mathbf{N}=80$.

\begin{tabular}{|c|c|c|c|c|c|}
\hline & \multicolumn{5}{|c|}{ power density } \\
\hline & $\mathbf{0}$ & $15 \mu \mathrm{W} / \mathrm{cm}^{2}$ & $31 \mu W / \mathrm{cm}^{2}$ & $1 \mathrm{~mW} / \mathrm{cm}^{2}$ & $5 \mathrm{~mW} / \mathrm{cm}^{2}$ \\
\hline Area $\left(\mu \mathrm{m}^{2}\right)$ & $42.59 \pm 5.14$ & $42.72 \pm 4.91$ & $42.99 \pm 5.03$ & $40.46 \pm 4.88^{*}$ & $40.73 \pm 4.99^{*}$ \\
\hline Diameter $(\mu \mathrm{m})$ & $7.04 \pm 0.45$ & $7.05 \pm 0.48$ & $7.09 \pm 0.44$ & $6.91 \pm 0.40^{*}$ & $6.86 \pm 0.43^{*}$ \\
\hline Long-short axis ratio & $1.03 \pm 0.05$ & $1.03 \pm 0.05$ & $1.03 \pm 0.05$ & $1.05 \pm 0.06^{*}$ & $1.06 \pm 0.05^{*}$ \\
\hline Normalized factor & $0.76 \pm 0.06$ & $0.76 \pm 0.05$ & $0.76 \pm 0.06$ & $0.79 \pm 0.04^{*}$ & $0.80 \pm 0.05^{*}$ \\
\hline
\end{tabular}

${ }^{*} \mathrm{P}<0.05 ; \mathrm{N}$ : number of data point in each measure.

Table 2. Geometric parameters of RBC vs irradiation time (for $900 \mathrm{MHz} \mathrm{EM}$ wave, $5 \mathrm{~mW} / \mathrm{cm}^{2}$ ) $\mathrm{N}=80$.

\begin{tabular}{|c|c|c|c|c|c|c|c|}
\hline$t$ (min) & $\mathbf{0}$ & 10 & 20 & 30 & 60 & 90 & 120 \\
\hline Area $\left(\mu \mathrm{m}^{2}\right)$ & $42.95 \pm 5.11$ & $42.88 \pm 5.05$ & $41.36 \pm 4.83^{*}$ & $40.55 \pm 4.70^{*}$ & $39.58 \pm 4.83^{\#}$ & $39.33 \pm 4.80$ & $39.19 \pm 4.92$ \\
\hline Diameter $(\mu \mathrm{m})$ & $7.05 \pm 0.46$ & $7.05 \pm 0.43$ & $6.92 \pm 0.50^{*}$ & $6.86 \pm 0.52^{*}$ & $6.78 \pm 0.43^{\#}$ & $6.80 \pm 0.40$ & $6.79 \pm 0.46$ \\
\hline Normalized factor & $0.75 \pm 0.06$ & $0.75 \pm 0.05$ & $0.78 \pm 0.06^{*}$ & $0.79 \pm 0.06^{*}$ & $0.82 \pm 0.05^{\#}$ & $0.82 \pm 0.06$ & $0.82 \pm 0.05$ \\
\hline
\end{tabular}

${ }^{*} \mathrm{P}<0.05$; $\mathrm{P}<0.01$; N: number of data point in each measure. 


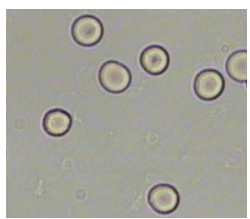

(a)

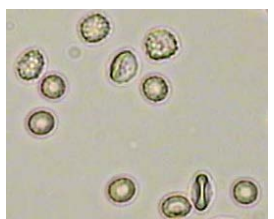

(b)

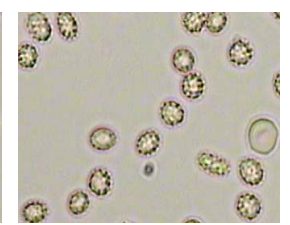

(c)
Figure 2. EM wave (900 $\left.\mathrm{MHz}, 5 \mathrm{~mW} / \mathrm{cm}^{2}\right)$ induced red cell deformation: (a) Before radiation; (b) After 30 minutes radiation; (c) After 60 minutes radiation.

perficial charge density after a period of EM wave radiation. These changes cause deformation and aggregation of RBCs and lead to an increase of blood density. Generally speaking, the change in long-short axis ratio reflects the extent of cells deformation; the change in normalized factors relates to deformation ability and blood liquidity, etc. The larger the normalized factors, the weaker the deformation ability and blood liquidity. The functions of RBC are generally related to their mechanical shapes. If the RBCs' morphology is abnormal, the superficial energy and mechanical characteristics of RBCs will change accordingly [14]. If the superficial charge density of RBCs decreases, cell aggregation and blood density may increase. The blood density and cell aggregation are positively correlated. The change in mechanical shape of RBCs will bring about the change in blood hemorrheology, which may increase. The resistance of blood flow leads to the block of capillaries and micro-veins. Due to insufficient blood flow and oxygen, some tissues may show acidosis, further red cell aggregation, deformability decrease, even vicious circle [15].

\section{Conclusions}

RBC is an important basic object in cell biology research, the study on biological effects of EM wave irradiation on RBCs will help understanding the biological effects of the cells with complex structure under irradiation. Many studies on the effect of EM wave on organisms are also targeted at cells, through which we can explore the role of the EM wave to individual organisms and then understand the EM radiation hazards and protection [16,17], also it would be helpful for us to develop standards of environmental EM radiation.

In this work, self-developed multi-dimensional microscopic imaging technique was used to monitor the immediate effects of the radio-frequency EM waves on the living RBCs. The different morphology parameters of RBCs were measured. It has been found that the effects of low-power density of EM irradiation on the the RBCs were dependent not only on the irradiation dose, but also on the irradiation time. Relevant effects happen only when the irradiation time is longer than a certain value, a time threshold. That indicates the EM effect on the cell could be cumulative. This study enriches the information of physical, biochemical and biological effects of radiofrequency EM waves on the RBC, and provides a new approach to study the bio-physical response of single living cell to EM radiation.

\section{Acknowledgments}

This work was supported by Science and Technology Program of Guangdong Province (NO:2011B031700061), Natural Science Foundation of Guangdong Province (NO:S20110 10004873), Scientific research project of Guangdong Pharmaceutical University (NO: 2007YGY02), and partially supported by Natural Science Foundation of Guangxi Province (NO: 2012GXNSFBA053001).

\section{REFERENCES}

[1] S. Velizarov, P. Raskmark and S. Kwee, "The Effects Radiofrequency Fields on Cell Proliferation Are Non-Thermal,” Bioelectrochem Bioenergetics, Vol. 48, 1999, pp. 177-180. http://dx.doi.org/10.1016/S0302-4598(98)00238-4

[2] M. Donnellan, D. R. McKenzie and P. W. French, "Effects of Exposure to Electromagnetic Radiation at 835 $\mathrm{MHz}$ on Growth, Morphology and Secretory Characteristics of a Mast Cell Analogue, RBL-2H3," Cell Biology International, Vol. 21, 1997, pp. 427-439. http://dx.doi.org/10.1006/cbir.1997.0162

[3] S. Pacini, M. Ruggiero, I. Sardi, S. Aterini, F. Gulisano and M. Gulisano, "Exposure to Gloal System for Mobile Communication(GSM) Cellular Phone Radiofrequency Alters Gene Expression, Proliferation and Morphology of Human Skin Fibroblasts,” Oncology Research, Vol. 13, 2002, pp. 19-24.

[4] M. Mashevich, D. Folkman, A. Kesar, A. Barbul, R. Korenstein, E. Jerby and L. Avivi, "Exposure to Human Peripheral Blood Lymphocytes to Electromagnetic Fields Associated with Cellular Phones Leads to Chromosomal Instability,” Bioelectromagnetics, Vol. 24, 2003, pp. 8290. http://dx.doi.org/10.1002/bem.10086

[5] D. Weisbrot, H. Lin, L. Ye, M. Blank and R. Goodman, "Effects of Mobile Phone Radiation on Reproduction and Development in Drosophila Melanogaster," Journal of Cellular Biochemistry, Vol. 89, 2003, pp. 48-55. http://dx.doi.org/10.1002/jcb.10480

[6] J. Czyz, K. Guan, Q. Zeng, T. Nikolova, A. Meister, F. Schonborn, J. Schuderer, N. Kuster and S. M. Wobus, "High Frequency Electromagnetic Fields (GSM Signals) Affect Gene Expression Levels in Tumour Suppressor p53-Deficient Embryonic Stem Cells,” Bioelectromagnetics, Vol. 25, 2004, pp. 296-307.

http://dx.doi.org/10.1002/bem.10199

[7] Z. Niu, H. B. Wang, J. Q. Hou, J. Yan and Z. Y. Lu, “Basic Theory Effects of Electromagnetic Wave on Ion Concentration in Cells," Chinese Journal of Biomedical Engineering, Vol. 21, 2002, pp. 552-556.

[8] M. Mashevich, D. Folkman, A. Kesar, A. Barbul, R. Korenstein, E. Jerby and L. Avivi, "Exposure of Human Peri- 
pheral Blood Lymphocytes to Electromagnetic Fields Associated with Cellular Phones Leads to Chromosomal Instability,” Bioelectromagnetics, Vol. 24, 2003, pp. 82-90. http://dx.doi.org/10.1002/bem.10086

[9] J. Tao, "Micro-Spectrophotometry \& Image Analysis with Applications in Structure \& Function Simultaneous Measurement on Single Intact Cell [D]," Sun Yat-sen University of Medical Sciences, Guangzhou, 2001, pp. 42-57 (in Chinese).

[10] L. L. Kang, Y. X. Huang, M. Luo, X. E. Yu and Z. J. Yuan, "Confocal Raman Micro-Spectroscopy of Lymphocyte Under Exposure to $900 \mathrm{MHz}$ Electromagnetic Fields," Journal of the Fourth Military Medical University, Vol. 28, No. 20, 2007, pp. 1862-1864 (in Chinese).

[11] C. S. Yao, "Instant Effects of $900 \mathrm{MHz}$ Electromagnetic Wave Radiation and Some Physical and Chemical Factors on Human Red Blood Cell. [D],” Sun Yat-sen University of MedicalSciences, Guangzhou, 2004 (in Chinese).

[12] C. C. Yao, Y. X. Huang, X. K. Li and P. Ruan, "Effects of $\mathrm{pH}$ on Structure and Function of Single Living Erythrocyte,” Chinese Science Bulletin, Vol. 48, 2003, pp. 1342-1346.

[13] C. C. Yao, P. Yao, G. H. Tang, Q. Zheng, X. K. Li and Y.
X. Huang, "Effects of Radio-Frequency Electromagnetic Wave and Environmental Temperature on Membrane Mechanical Properties of Single Living Intact Human Red Blood Cell,” China's Occupational Medicine, Vol. 32, 2005, pp. 11-13.

[14] P. Ruan, Y. X. Huang, M. Tu and S. H. Lin, “Change of the Dynamic Characteristics of Membrane and Oxygen Carrying Ability of the Erythrocyte in Different pH," Journal of the Fourth Military Medical University, Vol. 24, 2003, pp. 2119-2121.

[15] P. Ruan, Y. X. Huang and D. Li, "Spectrum Analysis of Erythrocyte Intracellular Hemoglobin with a Novel Fast Multi-Channel Micro-Spectrophotometry,” Spectroscopy and Spectral Analysis, Vol. 25, 2005, pp. 1121-1124.

[16] J. Fang, X. G. Huang, L. Ma, Y. Li and Y. J. Cao, "Effects of $900 \mathrm{MHz}$ Electromagnetic Field on DNA and the Protein Expression of p53 Gene in Human Embryonic Lung Cells,” Journal of Environment and Health, Vol. 24, 2007, pp. 251-253.

[17] Y. J. Zhou and Z. Q. Niu, "Mechanism of Biological Effects of ELF Pulsed Electromagnetic Wave on Cells," Space Medicine \& Medical Engineering, Vol. 21, 2008, pp. 121-125. 